\title{
Factors Associated with High Rates of Recurrence of Amebic Liver Abscess (ALA) in North India
}

\author{
Aradhana Singh, ${ }^{1}$ Tuhina Banerjee, ${ }^{1 *}$ and Sunit Kumar Shukla ${ }^{2}$ \\ ${ }^{1}$ Department of Microbiology, Institute of Medical Sciences, Banaras Hindu University, Varanasi, India; ${ }^{2}$ Department of Gastroenterology, Institute \\ of Medical Sciences, Banaras Hindu University, Varanasi, India
}

\begin{abstract}
Recurrence of amebic liver abscess (ALA), once considered unusual, is increasingly being reported, despite proper management. Realizing the endemicity of ALA in the study setup, this 2-year follow-up study was conducted to investigate the recurrent cases and study the associated factors. A total of 101 confirmed cases of ALA were followed up for a period of 2 years. Recurrent cases were studied for associated bacterial flora, presence of resistance genes (nim), level of matrix metalloproteinase 3 and MMP-9, and genotypes of Entamoeba histolytica and statistically compared with the nonrecurrent cases as controls. Recurrence rates of $8.9 \%$ (nine patients) were detected. The presence of Prevotella along with an increased level of MMP-9 in abscess fluid and large size of abscesses $(11 \times 10.8 \mathrm{~cm})$ was found to be significantly associated with recurrence in ALA. Among the nine cases, the presence of nimE gene was detected in two $(22.2 \%)$ patients. The genotyping of $E$. histolytica strains showed that in seven $(77.7 \%)$ cases, the genotype of E. histolytica was the same in the primary and recurrent samples. This study reports a high rate of recurrence in the cases of ALA, hinting toward the gradual development of clinical resistance toward the commonly used drug. The presence of nim gene and Prevotella in abscess fluid along with increased MMP-9 levels and large abscess size could be important predictors of recurrent ALA.
\end{abstract}

\section{INTRODUCTION}

Amebic liver abscess (ALA) is the most common extraintestinal manifestation of Entamoeba histolytica (E. histolytica) infections and is highly endemic in tropical countries including India. ${ }^{1}$ With an annual incidence of approximately 50 million cases of invasive $E$. histolytica infections worldwide, resulting in nearly 100,000 deaths, the parasite is a significant cause of morbidity in developing countries. ${ }^{2}$ There has been no recent global estimation on the epidemiology of this parasite and its varied presentation. There has been a decrease in the mortality because of ALA over the last decade. This decrease has been largely attributed to the effectiveness of antiprotozoal treatment. At the same time, there has been a significant increase in the prevalence of ALA cases. ${ }^{1}$

Recurrence in ALA is uncommon, ${ }^{3}$ and metronidazole remains the drug of choice for over 40 years. After appropriate treatment, it usually resolves and disappears completely. Large abscesses often require the use of combination therapy (percutaneous needle aspiration and pharmacotherapy). ${ }^{4}$ Acquired resistance to metronidazole has not been that frequent even after extensive use. ${ }^{5}$ Nonetheless, there have been intermittent reports of relapse and treatment failures in ALA patients following appropriate management. ${ }^{6-8}$ Resistance to 5-nitroimidazole drugs (metronidazole) is frequently linked with the presence of nitroimidazole reductase enzyme encoded by nim gene, which modulates the pathway of formation of the nitroso radicals that are critical for antimicrobial activity. ${ }^{9}$

Sporadic case studies on recurrent ALA have been reported from non-endemic regions like the United States, Mexico, and the Netherlands in the last 20 years. ${ }^{6,7}$ All such reports mention the visit of the respective cases to the endemic countries as the reason for recurrence. However, because of lack of published long-term follow-up reports of ALA patients, the frequency of recurrent ALA in endemic settings is not known.

*Address correspondence to Tuhina Banerjee, Department of Microbiology Institute of Medical Sciences, Banaras Hindu University, Lanka Varanasi, 221005 India. E-mail: drtuhina@yahoo.com
In ALA, the parasite causes immense extracellular matrix (ECM) destruction, and matrix metalloproteinases (MMPs) are reported to be the main enzymes involved in ECM degradation. ${ }^{10}$ Metalloproteinases are over expressed in a number of infections with protozoan parasites ${ }^{11}$ including amebic colitis. $^{12}$ The parasite E. histolytica uses its virulence factor (cysteine proteinase, CP-A5) along with the human MMPs for ECM degradation and to invade colonic tissues. ${ }^{12}$ However, any data regarding involvement of these MMPs in ALA are not available.

Amebic liver abscess has also been found to be prevalent in the neighboring country of Bangladesh, especially in those districts located in the Ganga basin. ${ }^{13}$ In one of our recent studies, considerably high prevalence of ALA (87\%) among liver abscess cases has been detected. ${ }^{1}$ As the study population and location of the present report are also along the Ganga river basin, we hypothesized that there could be a higher rate of recurrence of ALA in this region. With this background, this preliminary study was carried out to investigate the recurrent cases of ALA and the association with anaerobic microflora, genotypes, resistance genes (nim) and matrix metalloproteinase 3 (MMP-3), and MMP-9 for better assessment of the situation.

\section{METHODS}

This was a prospective cross-sectional study conducted in the department of microbiology in collaboration with the department of gastroenterology of a tertiary care university hospital in Varanasi, India. The study was ethically approved by an institute ethical committee (Dean/2016-17/EC/045). Informed consent was obtained from all the patients before the study.

The study included 101 confirmed cases of ALA, who were tested positive by nested multiplex PCR for E. histolytica. The criteria for including these cases were that all these cases were primary cases of ALA without previous history and records of any similar abscesses. Those cases associated with complications and comorbidities such as hypertension, diabetes, and malignancy, and so on were excluded. All the cases included in the study were followed up at regular intervals of 1 month for a period of the first 6 months after their first visit 
and then at intervals of 3 months for 2 years. At every followup, the cases were screened for response to treatment by repeat ultrasonography to detect the size of the abscess along with detailed history. Besides, liver function tests and leukocyte count were also performed to assess response to treatment at each follow-up. Recurrence was defined as those cases who had initially responded to treatment by complete resolution of the abscess confirmed through ultrasonography and laboratory tests but had subsequently developed ALA during the study period.

Aspirates from the abscesses were aseptically collected through ultrasound-guided percutaneous needle aspiration and immediately transferred to the laboratory, where they were subjected to microscopy and detection of $E$. histolytica by nested multiplex PCR targeting E. histolytica, Entamoeba dispar, and Entamoeba moshkovskii as per reference. ${ }^{14}$ Bacterial flora was studied by inoculating the aspirates on blood agar and MacConkey agar, followed by overnight incubation at $37^{\circ} \mathrm{C}$. The presence of relevant anaerobes in the aspirates was detected by conventional PCR using genus-specific primers of Peptococcus, Peptostreptococcus, Prevotella, Clostridium, Fusobacterium, Bifidobacterium, and Bacteroides. ${ }^{15}$

The cases that showed recurrence during the study period was further analyzed for the various genotypes of $E$. histolytica, presence of nim genes, and levels of MMP-3 and MMP-9 in the aspirates. The detection of nim genes was carried out by conventional PCR using the universal set of primers, Nim-3 and Nim-5, for all known nim genes. ${ }^{16}$ The reaction conditions included an initial denaturation at $94^{\circ} \mathrm{C}$ for 10 minutes, followed by 32 cycles of denaturation at $94^{\circ} \mathrm{C}$ for 30 seconds, annealing at $62^{\circ} \mathrm{C}$ for 1 minute, extension at $72^{\circ} \mathrm{C}$ for 1 minute, and, at last, a final extension at $72^{\circ} \mathrm{C}$ for 10 minutes. The amplicons were further subjected to sequencing, and the sequences were analyzed using the BLASTN program for homology search within the existing database.

Levels of MMP-3 and MMP-9 in the liver aspirate samples were estimated using a human MMP ELISA kit (Boster biological technology co., Ltd, Pleasanton, CA) as per the manufacturer's instructions. The concentration of MMP-3 and MMP-9 protein in the test and control samples was calculated using GraphPad Prism 8.0.0.224 software (GraphPad Software, San Diego, CA).

For PCR-based genotyping of E. histolytica, amplification was performed with $E$. histolytica-specific primer pairs targeting six tRNA-linked polymorphic STR loci (A-L, D-A, N-K, $R-R, S^{T G A}-D$, and $\left.S-Q\right)$, as described elsewhere. ${ }^{17}$

Statistical analysis. A case-control analysis was performed considering all the patients who showed recurrence as cases and four times the number of randomly chosen patients from among those who did not show recurrence as controls. Factors such as demographic profile, socioeconomic conditions, hemoglobin levels, size of the abscess, associated anaerobic microflora, and levels of MMP-3 and MMP-9 were compared by Student's $t$ test for continuous variables and by the chi-square test for categorical variables using 2019 MedCalc software (version: bv) and SPSS version 19 (Armonk, NY). A $P$ value of $<0.05$ was considered as significant.

\section{RESULTS}

Of the total of 101 cases, nine patients (8.9\%) showed recurrence within the study period. All the recurrent cases were males of different ages ranging from 18 to 65 years, with a mean age of $43.3 \pm 13.4$ years. Seven $(77.7 \%)$ of the total subjects were chronic alcoholics and anemic. All the study patients were HIV negative. Table 1 shows the comparative details of all the cases and controls. All the cases with recurrence $(n=9)$ presented with the complaints of abdominal pain and fever with right-sided abdominal heaviness as the prominent symptoms at recurrence. The right lobe was the site of infection in all the cases, and the abscesses were larger than the average size of $11 \times 10.8 \mathrm{~cm}$. In four $(44.4 \%)$ of the total cases, the large size of the abscesses required pig-tail drainage treatment, instead of syringe aspiration. The duration between the primary and the recurrent cases varied from 28 weeks to 60 weeks. All the 101 ALA patients had received treatment with metronidazole $(750 \mathrm{~g})$ and Augmentin $(500 \mathrm{~g})$

TABLE 1

Socioeconomic, demographic, and clinical profiles of the cases and controls

\begin{tabular}{|c|c|c|c|c|c|c|}
\hline S. no. & Factors & & Cases $(n=9)$ & Control $(n=36)$ & Odds ratio $(95 \% \mathrm{Cl})$ & $P$-value \\
\hline \multirow[t]{2}{*}{1.} & Age (average $=42.5 \pm 13.3$ years) & $\geq$ Average & $4(44.4)$ & $18(50)$ & $0.8(0.18-3.47)$ & 0.765 \\
\hline & & $<$ Average & $5(55.5)$ & $18(50)$ & & \\
\hline \multirow[t]{2}{*}{2.} & Residence & Rural & $6(66.6)$ & $19(52.7)$ & $1.7(0.3-8.2)$ & 0.456 \\
\hline & & Urban & 3 (33.3) & $17(47.2)$ & & \\
\hline \multirow[t]{2}{*}{3.} & Water source & Untreated & $8(88.8)$ & $27(75)$ & $2.6(0.2-24.3)$ & 0.384 \\
\hline & & Treated & $1(11.1)$ & $9(25)$ & & \\
\hline \multirow[t]{2}{*}{4.} & Anemia & Present & $7(77.7)$ & $19(52.7)$ & $3.1(0.5-17.1)$ & 0.188 \\
\hline & & Absent & $2(22.2)$ & $17(47.2)$ & & \\
\hline \multirow[t]{2}{*}{5.} & Alcoholism & Present & 7 (77.7) & 25 (69.4) & $1.5(0.2-8.6)$ & 0.623 \\
\hline & & Absent & 2 (22.2) & $11(30.5)$ & & \\
\hline \multirow[t]{5}{*}{6.} & Common anaerobes ${ }^{\star}$ & Bacteroides & 0 & $3(8.3)$ & $0.5(0.02-10.6)$ & 0.659 \\
\hline & & Peptostreptococcus & 0 & $1(2.7)$ & $1.2(0.04-33)$ & 0.895 \\
\hline & & Prevotella & $5(55.5)$ & $4(11.1)$ & $10(1.87-53.4)$ & $0.007 \dagger$ \\
\hline & & Peptococcus & 0 & $6(16.6)$ & $0.24(0.01-4.8)$ & 0.355 \\
\hline & & Fusobacterium & 0 & $6(16.6)$ & $0.24(0.01-4.8)$ & 0.355 \\
\hline \multirow[t]{2}{*}{7.} & MMP -9 concentration (average $=$ & $\geq$ Average & $6(66.6)$ & $9(25)$ & $6.0(1.23-29.07)$ & $0.026 \dagger$ \\
\hline & $7,878.46 \mathrm{pg} / \mathrm{mL})$ & $<$ Average & $3(33.3)$ & $27(75)$ & & \\
\hline 8. & $\begin{array}{l}\text { Matrix metalloproteinase } 3 \text { concentration } \\
\quad \text { (average }=21,890 \mathrm{pg} / \mathrm{mL} \text { ) }\end{array}$ & $\begin{array}{l}\geq \text { Average } \\
<\text { Average }\end{array}$ & $\begin{array}{l}2 \\
7\end{array}$ & $\begin{array}{c}9 \\
27\end{array}$ & $0.8571(0.15-4.8991)$ & 0.8624 \\
\hline \multirow[t]{2}{*}{9.} & Abscess size $($ average $=9.5 \times 9.3)$ & $\geq$ Average & $6(66.6)$ & $10(27.7)$ & $5.2(1.08-24.89)$ & $0.039 \dagger$ \\
\hline & & $<$ Average & $3(33.3)$ & $26(72.2)$ & & \\
\hline
\end{tabular}

${ }^{*}$ Bifidobacterium and Clostridium were also screened in cases and controls; however, they were not present in any of the screened samples.

$\dagger P$ value $<0.05$ (significant) 
twice daily for 10 days at their primary presentation. No death was recorded during the study period.

Detection of anaerobes revealed the presence of Prevotella in five $(55.5 \%)$ of the recurrent cases. However, in the control group, Peptococcus (16.6\%) and Fusobacterium (16.6\%) were found to be the most prevalent anaerobes. The concentration of MMP-3 protein did not show any significant difference in the test group and control group. However, the concentration of MMP-9 protein in the test group was 3.0-fold higher than that in the control group. Abscess fluid MMP-9 showed a positive relation with the abscess size in the present study. By comparison, the cases of recurrence were significantly $(P<0.05)$ associated with the presence of Prevotella in the ALA fluids, increased levels of MMP-9, and large size of abscesses.

Among the nine cases of recurrence, the presence of nim genes was detected in two (22.2\%) patients (Figure 1). None of the control samples showed the presence of nim genes. The sequence analysis confirmed the presence of nimE gene in both the cases. The genotyping of $E$. histolytica strains based on six tRNA-linked polymorphic STR loci showed that in seven $(77.7 \%)$ of the total recurrent cases, the genotype of $E$. histolytica was the same in the primary and recurrent samples (Figure 2). However, in two cases, a new genotype was detected, showing polymorphism at N-K, S-Q, and D-A loci, which could be the cases of reinfection. Maximum polymorphism was seen at the D-A locus both in the case and control groups.

\section{DISCUSSION}

Amebic liver abscess caused by the protozoan parasite $E$. histolytica is a major public health problem in developing countries like India. There are currently no reports of resistance to commonly used antimicrobial agents, but reports of recurrent ALA and clinical treatment failures are on rise. This prompted us to investigate the recurrence in ALA cases in one of the endemic regions.

This study revealed a recurrence rate of $8.9 \%$ among the primary cases of ALA within a period of 2 years. Individual case reports are on record from India in the recent past where multiple relapses of ALA have been reported, despite metronidazole treatment. ${ }^{18} \mathrm{~A}$ recurrence rate of $6.7 \%$ has been previously reported from Bangladesh in a similar 2-year follow-up study. ${ }^{13}$ Among the other sporadic reports of recurrence, one study revealed the case of an individual developing three episodes of ALA in a period of 4 years in which the second episode of ALA was seen 18 months after the first one and the third appeared 12 months after the second one. ${ }^{6}$ Cases of "re-incidence" without travel to endemic areas from nonendemic countries like France have also been reported even after treatment with adequate amebicidal agents. ${ }^{19}$ A study of 152 cases of hepatic amebiasis also demonstrated relapse in four patients at mid- or long term after apparent recovery, in the absence of any noticeable reinfection. ${ }^{20}$

A relapse rate of $10 \%$ has been seen in the past in the cases of ALA with only metronidazole therapy and in the absence of luminal agents like paromomycin. This might be because of the survival of the parasites in the lumen of the colon. ${ }^{21}$ However, a study conducted almost 35 years back in France to evaluate the status of invasive amebiasis in retropositive patients showed recurrence of invasive amebiasis in 12 (7\%) cases during the entire follow-up period, irrespective of the use of any luminal agent. ${ }^{22}$

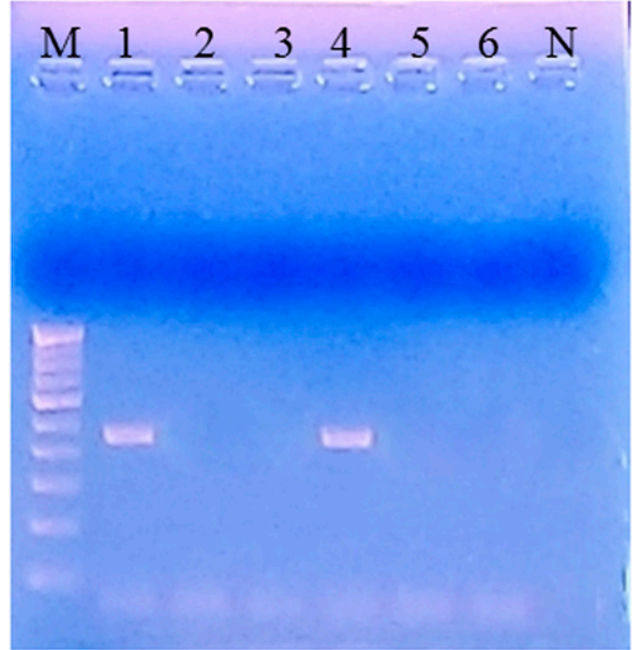

Lane M: Ladder (100bp), Lane

$$
\begin{aligned}
& \text { 1,4: Samples positive for nim } \\
& \text { gene, Lane 2,3,5,6: Samples } \\
& \text { negative for nim gene, Lane N: } \\
& \text { Negative control. }
\end{aligned}
$$

FIGURE 1. Agarose gel electrophoresis showing the presence of nim genes in the liver aspirate samples of recurrent cases under a ultra-violet transilluminator. This figure appears in color at www.ajtmh.org.

Recurrent ALA has been described to be more common with factors such as alcohol, corticosteroids, and immunosuppression. ${ }^{18}$ This is in concordance with this study as majority (7/9) of the patients were chronic alcoholics. The duration between the first and second liver abscess varied from 28 to 60 weeks, having a mean duration of $41 \pm 11.3$ weeks. Previous reports have shown the frequency of recurrence of ALA ranging from three times over the duration of 16 years $^{3}$ to thrice in a year. ${ }^{23}$ As cases were followed up for 2 years in the present study, further evaluation of the course of the infection in the recurrent cases could not be carried out.

Management of ALA usually involves the use of tissue amebicides, mostly metronidazole, with or without aspiration of the cavity. Based on earlier studies, combination therapy is

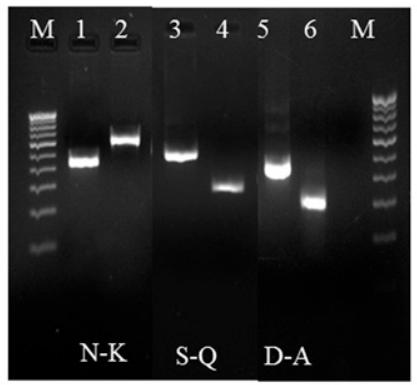

M:100 bp ladder. Lane 1,3,5: STR's loci in the primary aspirate, Lane 2,4,6: STR's loci in the recurrent aspirate.

FIGURE 2. Different sizes of short tandem repeats (STRs) at N-K, S-Q, and $D-A$ loci indicating the case of new infection in the recurrent case. 
preferred in this study center for management of ALA with a size greater than $5 \mathrm{~cm} .{ }^{4}$ Other luminal agents were not used and therefore could not be studied. Metronidazole is the major drug of choice in the case of amebiasis. Any known resistance to this drug has not been reported. However, recent reports of variations in drug susceptibilities are on record, indicating that a minor percentage of ameba is either resistant or eventually will become resistant because of indiscriminate use of antiamebic drugs. ${ }^{5}$ In vitro drug susceptibility testing is often cumbersome and inaccurate owing to the requirement of axenically grown parasites, which often exhibit phenotypic changes. Similarly, monoxenic and polyxenic cultures can confound the susceptibility readings because of the overgrowth of associated microflora in the culture. ${ }^{24}$

Metronidazole is often used irrationally as an empirical therapy for all types of diarrhea in developing countries. ${ }^{25}$ Metronidazole resistance is often linked to the nim genes. In the last decade, the presence of nim gene has been associated with the resistance against metronidazole in many anaerobes, such as Bacteroides, Peptostreptococcus, and Prevotella. Till date, $11 \mathrm{nim}$ genes, $\operatorname{nimA}$ to $\operatorname{nimK}$, have been reported ${ }^{9}$ from Bacteroides and Prevotella species which are either plasmid- or chromosomally encoded. Lateral transfer of the resistance gene of metronidazole has also been seen. ${ }^{26}$ In this study, two (22.2\%) pus samples from ALA showed the presence of nimE gene. A previous study has shown that metronidazole challenge might cause rapid amplification of nim genes in the population. ${ }^{27}$ Besides, as studies have documented differences in the presence of nim genes in the microflora of amebic and hospitalized patients as against healthy individuals, ${ }^{28,29}$ it could be presumed that these genes could have been responsible for inactivation of nitroimidazoles used in treatment, thus facilitating persistence.

Entamoeba histolytica trophozoites coexist with varying bacterial populations, and anaerobes are majorly associated with this parasite. ${ }^{1}$ In this study, we found Prevotella to be specifically associated with more than half of the recurrent cases. Previous literature also suggests that Prevotella has been associated with the enhanced gut inflammation and confirmed that high parasite burden and expansion of Prevotella levels were associated with the diarrhea due to E. histolytica. ${ }^{30}$ Also, a cross-sectional study conducted in Limpopo Province of South Africa showed higher Prevotella burden in cases of diarrhea due to $E$. histolytica than nondiarrheal patients. ${ }^{31}$ These findings hint toward enhancement of the E. histolytica virulence in the presence of Prevotella, which could account for persistent infections. Predominance of Prevotella in recurrent ALA cases has never been reported before this study.

All abscesses included in the test group in this study were large in size $\left(>8 \mathrm{~cm}^{2}\right)$. A previous study has shown a significant correlation between the length of the treatment and the size and number of the abscesses. ${ }^{32}$ Another study reported that the probability of the failure of percutaneous needle aspiration increases with the increase in the size of abscess cavity to be aspirated. ${ }^{33}$ Rapid re-accumulation of pus in the abscess can be a reason for failure of needle aspiration. ${ }^{34}$ This finding thus suggests that the size of the liver abscess can be a risk factor for the recurrence of ALA.

In ALA, the parasite causes massive destruction of the ECM with the help of MMPs. ${ }^{10}$ This study focused on MMP-3 and MMP-9 because they are the most widely investigated MMPs and their overexpression has been reported in diseased conditions. ${ }^{35}$ In this study, significantly higher levels of MMP-9 were detected in patients with recurrent $A L A$, and a positive correlation between the concentration of MMP-9 and the size of the abscess was detected. Therefore, the overexpression and activation of MMP-9 could contribute to the pathogenesis of ALA in recurrent cases. The up-regulation of the MMP-3 gene expression has been demonstrated in cases of intestinal invasion by $E$. histolytica trophozoites. ${ }^{12}$ However, no such association was detected for MMP-3 in the ALA samples in this study.

Majority of the follow-up samples from the recurrent cases showed the same genotype. In two cases, new genotype in the recurrent case was seen, indicating new infection. Repeated exposure to $E$. histolytica in endemic areas like the present study center often results in cyclic reinfection. ${ }^{6}$ In a study conducted on screening of intestinal amebiasis in children in Bangladesh, 23 follow-up samples were obtained, of which seven (30.4\%) showed the presence of new genotypes in the follow-up cases. ${ }^{36}$ No data are available in the literature on the genotypes of $E$. histolytica in recurrent ALA cases for commenting on this study finding. However, we must acknowledge that because the genotyping was performed from the DNA directly in the aspirate samples, there might be a possibility that DNA from the debris of the previous abscess affected the genotypes. This limitation was minimized because the abscesses showed healing in terms of reduction in size during the follow-up period, although genotyping from culture of the parasite would have been the best option.

This study has documented recurrent cases of ALA in an endemic setup. The exact underlying mechanism for recurrence in such cases remains unclear. However, the observations from the present study suggest that this could be an early hint of gradual development of clinical resistance to metronidazole among patients with ALA. The presence of nim genes and Prevotella in abscess fluid along with increased MMP-9 levels and large abscess size could be important predictors of recurrent ALA. The role of other luminal agents should be further evaluated in endemic settings. Similar studies of long-term duration on cases of ALA are required to validate these findings.

Received January 28, 2020. Accepted for publication November 25, 2020.

Published online January 11, 2021.

Acknowledgments: We thank Banaras Hindu University and the Department of Science and Technology (DST), India, for providing the facilities to conduct this study. The American Society of Tropical Medicine and Hygiene (ASTMH) assisted with publication expenses.

Financial support: This research did not receive any specific grant from funding agencies in the public, commercial, or not-for-profit sectors.

Authors' addresses: Aradhana Singh and Tuhina Banerjee, Department of Microbiology, Institute of Medical Sciences, Banaras Hindu University, Varanasi, India, E-mails: aradhanasingh.148@ gmail.com and drtuhina@yahoo.com. Sunit Kumar Shukla, Department of Gastroenterology, Institute of Medical Sciences, Banaras Hindu University, Varanasi, India, E-mail: sunitshuklabhu@gmail.com.

\section{REFERENCES}

1. Singh A, Banerjee T, Kumar R, Shukla SK, 2019. Prevalence of cases of amebic liver abscess in a tertiary care centre in India: a study on risk factors, associated microflora and strain variation of Entamoeba histolytica. PLoS One 14: e0214880. 
2. Dhawan VK, Cleveland KO, Cantey JR, 2019. Amebiasis Workup: Laboratory Studies, Radiography, Ultrasonography, CT, and MRI, Liver Aspiration. Available at: https://emedicine.medscape. com/article/212029-workup. Accessed October 23, 2019.

3. Creemers-Schild D, van Genderen PJJ, Visser LG, van Hellemond JJ, Wismans PJ, 2016. Recurrent amebic liver abscesses over a 16-year period: a case report. BMC Res Notes 9: 472.

4. Ghosh JK, Goyal SK, Behera MK, Tripathi MK, Dixit VK, Jain AK, Shukla R, 2016. Efficacy of aspiration in amebic liver abscess. Trop Gastroenterol 36: 251-255.

5. Bansal D, Sehgal R, Chawla Y, Mahajan RC, Malla N, 2004. In vitro activity of antiamoebic drugs against clinical isolates of Entamoeba histolytica and Entamoeba dispar. Ann Clin Microbiol Antimicrob 3: 27.

6. Ramiro M, Moran P, Olvera H, Curiel O, Gonzalez E, Ramos F, Melendro El, Ximénez C, 2000. Reincidence of amebic liver abscess: a case report. Arch Med Res 31: S1-S3.

7. Hwang EW, Cheung L, Mojtahed A, Cartwright CA, 2011. Relapse of intestinal and hepatic amebiasis after treatment. Dig Dis Sci 56: $677-680$.

8. Moran P, Rico G, Ramiro M, Olvera H, Ramos F, González E, Valadéz A, Curiel O, Melendro El, Ximénez C, 2002. Defective production of reactive oxygen intermediates $(\mathrm{ROI})$ in a patient with recurrent amebic liver abscess. Am J Trop Med Hyg 67: 632-635.

9. Alauzet C, Mory F, Teyssier C, Hallage H, Carlier JP, Grollier G, Lozniewski A, 2010. Metronidazole resistance in Prevotella spp. and description of a new nim gene in Prevotella baroniae. Antimicrob Agents Chemother 54: 60-64.

10. Piña-Vázquez C, Reyes-López M, Ortíz-Estrada G, De la Garza M, Serrano-Luna J, 2012. Host-parasite interaction: parasitederived and-induced proteases that degrade human extracellular matrix. J Parasitol Res 2012: 748206.

11. Geurts N, Opdenakker G, Van den Steen PE, 2012. Matrix metalloproteinases as therapeutic targets in protozoan parasitic infections. Pharmacol Ther 133: 257-279.

12. Thibeaux R, Ave P, Bernier M, Morcelet M, Frileux P, Guillen N, Labruyère $\mathrm{E}, 2014$. The parasite Entamoeba histolytica exploits the activities of human matrix metalloproteinases to invade colonic tissue. Nat Commun 5: 5142.

13. Alam F, Salam MA, Hassan P, Mahmood I, Kabir M, Haque R, 2014. Amebic liver abscess in northern region of Bangladesh: sociodemographic determinants and clinical outcomes. BMC Res Notes 7: 625.

14. Khairnar K, Parija SC, 2007. A novel nested multiplex polymerase chain reaction (PCR) assay for differential detection of Entamoeba histolytica, E. moshkovskii and E. dispar DNA in stool samples. BMC Microbiol 7: 47.

15. Rekha R, Alam Rizvi M, Jaishree P, 2006. Designing and validation of genus-specific primers for human gut flora study. Electron $J$ Biotechn 9: 505-511.

16. Trinh S, Reysset G 1996. Detection by PCR of the nim genes encoding 5-nitroimidazole resistance in Bacteroides spp. J Clin Microbiol 34: 2078-2084.

17. Ali IKM, Zaki M, Clark CG, 2005. Use of PCR amplification of tRNA gene-linked short tandem repeats for genotyping Entamoeba histolytica. J Clin Microbiol 43: 5842-5847.

18. Singal DK, Mittal A, Prakash A, 2012. Recurrent amebic liver abscess. Indian J Gastroenterol 31: 271-273.
19. Nespola B et al., 2015. First case of amebic liver abscess 22 years after the first occurrence. Parasite 22: 20.

20. Laverdant C, Denee JM, Roue R, Molinie C, Daly JP, Flechaire A, Valmary J, Farret O, 1984. Hepatic amebiasis: study of 152 cases. Gastroenterol clin Biol 8: 838-844.

21. Brailita DM, Lingvay I, Aung K, Ojha A, 2019. Amebic Liver/ Hepatic Abscesses Treatment and Management. Available at: https://emedicine.medscape.com/article/183920-treatment. Accessed November 14, 2020.

22. Watanabe K, Gatanaga H, Escueta-de Cadiz A, Tanuma J, Nozaki T, Oka S, 2011. Amebiasis in HIV-1-infected Japanese men: clinical features and response to therapy. PLoS Neg/Trop Dis 5: e1318.

23. Gregory PB, 1976. A refractory case of hepatic amoebiasis. Gastroenterology 70: 585-588.

24. Fotedar R, Stark D, Beebe N, Marriott D, Ellis J, Harkness J, 2007. Laboratory diagnostic techniques for Entamoeba species. Clin Microbiol Rev 20: 511-532.

25. Banerjee T, Anupurba S, Filgona J, Singh DK, 2015. Vancomycinresistance enterococcal colonization in hospitalized patients in relation to antibiotic Usage in a tertiary care hospital of North India. J Lab Physicians 7: 108-111.

26. Löfmark S, Fang H, Hedberg M, Edlund C, 2005. Inducible metronidazole resistance and nim genes in clinical Bacteroides fragilis group isolates. Antimicrob Agents Chemother 49: 1253-1256.

27. Verma AK, Verma R, Ahuja V, Paul J, 2012. Real-time analysis of gut flora in Entamoeba histolytica infected patients of Northern India. BMC Microbiol 12: 183.

28. Rani R, Murthy RS, Bhattacharya S, Ahuja V, Rizvi MA, Paul J, 2006. Changes in bacterial profile during amebiasis: demonstration of anaerobic bacteria in ALA pus samples. Am J Trop Med Hyg 75: 880-885.

29. Katsandri A, Avlamis A, Pantazatou A, Houhoula DP, Papaparaskevas J, 2006. Dissemination of nim-class genes, encoding nitroimidazole resistance, among different species of Gram-negative anaerobic bacteria isolated in Athens, Greece. $J$ Antimicrob Chemother 58: 705-706.

30. Gilchrist CA et al., 2015. Role of the gut microbiota of children in diarrhea due to the protozoan parasite Entamoeba histolytica. $J$ Infect Dis 213: 1579-1585.

31. Ngobeni R, Samie A, Moonah S, Watanabe K, Petri WA, Jr., Gilchrist C, 2017. Entamoeba species in South Africa: correlations with the host microbiome, parasite burdens, and first description of Entamoeba bangladeshi outside of Asia. J Infect Dis 216: 1592-1600.

32. DeWitt J, Reining A, Allsworth JE, Peipert JF, 2010. Tuboovarian abscesses: is size associated with duration of hospitalization \& complications? Obstet Gynecol Int 2010: 847041.

33. Bansal A, Bansal AK, Bansal V, Kumar A, 2016. Liver abscess: catheter drainage $\mathrm{v} / \mathrm{s}$ needle aspiration. Int Surg J 2: 20-25.

34. Dietrick RB, 1984. Experience with liver abscess. Am J Surg 147: 288-291.

35. Gu ZD, Chen KN, Li M, Gu J, Li JY, 2005. Clinical significance of matrix metalloproteinase-9 expression in esophageal squamous cell carcinoma. World J Gastroenterol 11: 871-874.

36. Ali IK, Mondal U, Roy S, Haque R, Petri WA, Clark CG, 2007. Evidence for a link between parasite genotype and outcome of infection with Entamoeba histolytica. J Clin Microbiol 45: 285-289. 